\title{
Comprehensive Cancer Panel Sequencing Defines Genetic Diversity and Changes in the Mutational Characteristics of Pancreatic Cancer Patients Receiving Neoadjuvant Treatment
}

\author{
Kyong-Ah Yoon ${ }^{1}$, Sang Myung Woo ${ }^{2,3}$, Yun-Hee Kim ${ }^{3}$ Sun-Young Kong, ${ }^{3,4}$ Min Kyoung Lee ${ }^{3}$, Sung-Sik Han ${ }^{2}$, Tae Hyun Kim², \\ Woo Jin Lee ${ }^{2}$, and Sang-Jae Park ${ }^{2}$ \\ ${ }^{1}$ College of Veterinary Medicine, Konkuk University, Seoul, ${ }^{2}$ Center for Liver Cancer, Hospital, National Cancer Center, ${ }^{3}$ Department of Cancer \\ Biomedical Science, National Cancer Center Graduate School of Cancer Science and Policy, and ${ }^{4}$ Center for Diagnostic Oncology, Hospital, \\ National Cancer Center, Goyang, Korea
}

Background/Aims: Pancreatic ductal adenocarcinoma (PDA) is associated with an extremely poor prognosis. This study assessed the genetic diversity among patients with PDA and compared their mutational profiles before and after treatment. Methods: Tumors and matched blood samples were obtained from 22 PDA patients treated with neoadjuvant chemoradiation therapy. The somatic mutations were analyzed with comprehensive cancer gene panel (CCP). In addition, the biopsy samples obtained at diagnosis and the surgically resected samples after treatment were compared for seven patients. The CCP provided formalin-fixed paraffinembedded sample-compatible multiplexed target selection for 409 genes implicated in cancer. Results: Assessments of the MLH1, MLH3, MSH2, and PMS2 genes showed that the four patients with the highest relative burdens of mutations harbored somatic mutations in at least three of these genes. Genes in the histone-lysine N-methyltransferase 2 (KMT2) family, such as KMT2D, KMT2A, and KMT2C, were frequently mutated in tumor samples. Survival was worse in patients with $A R I D 1 A$ gene mutations than those without $A R I D 1 A$ gene mutations. Mutation patterns were compared between tissue samples before and after neoadjuvant treatment in seven patients who underwent surgical resection. The allelic fraction of mutations in KRAS codon 12 was lower in the surgically resected samples than in the endoscopic ultrasonography-guided fine needle aspiration biopsy samples of six patients. The number of mutant alleles of the histone lysine methyltransferase gene WHSC1 also decreased after treatment. Conclusions: These results indicate that tumor tissue from PDA patients is genetically diverse and suggest that $A R I D 1 A$ mutations may be a potential prognostic marker for PDA. (Gut Liver 2019;13:683-689)

Key Words: Pancreatic neoplasms; ARID1A; Histone-lysine Nmethyltransferase

\section{INTRODUCTION}

Pancreatic cancer is the fifth most common cancer among Korean males, with a steadily increasing incidence rate in recent decades in Korea. ${ }^{1,2}$ At present, the crude annual incidence of pancreatic cancer in Korea is approximately 10.9 per 100,000 persons. Pancreatic ductal adenocarcinoma (PDA) is the predominant histologic type, accounting for about $85 \%$ of pancreatic cancers. Risk factors for pancreatic cancer include chronic pancreatitis, heavy smoking, and obesity, but there is no effective screening tool for early diagnosis. ${ }^{3}$

Pancreatic cancer is also one of the top five causes of cancer deaths worldwide, as well as having the lowest 5-year survival rate among solid tumors. In Korea, the 5-year survival rate of both men and women with pancreatic cancer between 2010 and 2014 was only 10.1\%. ${ }^{1}$ Current treatments for PDA include a combination of gemcitabine and nab-paclitaxel, or a combination of fluorouracil, irinotecan, oxaliplatin, and leucovorin (FOLFIRINOX). ${ }^{4,5}$ Although several clinical trials have shown that these treatments improve survival among patients with PDA, the effects of treatment vary. Genetic features of PDA may help identify targets for treatment, as well as genetic markers associated with patient prognosis, thereby providing clinical benefits for patients with PDA.

\footnotetext{
Correspondence to: Sang Myung Woo ${ }^{\mathrm{a}}$ and Sang-Jae Park ${ }^{\mathrm{b}}$

Center for Liver Cancer, Hospital, National Cancer Center, Ilsan-ro, Ilsandong-gu, Goyang 10408, Korea

${ }^{a}$ Tel: +82-31-920-1733, Fax: +82-31-920-2798, E-mail: wsm@ncc.re.kr

'Tel: +82-31-920-1640, Fax: +82-31-920-1640, E-mail: spark@ncc.re.kr Received on August 8, 2018. Revised on December 18, 2018. Accepted on December 26, 2018. Published online June 28, 2019. pISSN 1976-2283 eISSN 2005-1212 https://doi.org/10.5009/gnl18355

(a) This is an Open Access article distributed under the terms of the Creative Commons Attribution Non-Commercial License (http://creativecommons.org/licenses/by-nc/4.0) which permits unrestricted non-commercial use, distribution, and reproduction in any medium, provided the original work is properly cited.
} 
Activating mutations of the KRAS gene are frequently detected in PDAs, with these mutations considered a genetic factor associated with poor prognosis. ${ }^{6,7}$ Genetic studies have reported the inactivation of tumor suppressor genes, such as TP53, $C D$ $K N 2 A$, and SMAD4, in more than 50\% of PDAs. ${ }^{8,9}$ In addition, SWI/SNF-mediated chromatin remodeling, including alterations in the ARID1A, KDM6A and SMARCA2 genes, has emerged as the basis for additional pathways affected by somatic mutations in PDA. ${ }^{10,11}$

Genome analyses of PDAs have revealed considerable genetic diversity and a complex mutational landscape, making it difficult to determine genetic features associated with patient prognosis. However, the clinical value of serial monitoring of mutation profiles in PDA is unknown. Mutation patterns affected by treatment may help identify treatment targets. Genetic characteristics defined by a comprehensive cancer gene panel (CCP) have been used to predict clinical benefits with an accuracy similar to that of whole exome sequencing. ${ }^{12-14}$ This study assessed genetic diversity among patients with PDA and compared the mutational profiles before and after treatment.

\section{MATERIALS AND METHODS}

\section{Patients and collection of clinical data}

Tumor samples were obtained from surgically resected blocks from 14 patients who underwent preoperative chemoradiotherapy with gemcitabine and eight patients who received induction chemotherapy and chemoradiotherapy. All of these patients had been enrolled in two phase II clinical trials at the National Cancer Center of Korea (ClinicalTrials.gov numbers: NCT01333124 and NCT01593475, respectively). These trials were started in 2012, and their protocols were approved by the Institutional Review Board of the National Cancer Center of Korea (IRB numbers: NCCCTC-10-500 and NCCCTC-10-567, respectively). Written informed consents were obtained.

Fourteen patients with resectable pancreatic cancer received gemcitabine $400 \mathrm{mg} / \mathrm{m}^{2}$ as an intravenous 30-minute infusion on days 1, 8, 15, 22, and 29, along with radiotherapy. Eight patients with locally advanced pancreatic cancer received induction chemotherapy, consisting of gemcitabine $(1,000 \mathrm{mg} /$ $\mathrm{m}^{2}$ ) and cisplatin $\left(25 \mathrm{mg} / \mathrm{m}^{2}\right)$ as intravenous infusions on days 1,8 and 15 of each 28-day treatment cycle. ${ }^{15}$ Within 3 weeks of completing two cycles of induction chemotherapy, patients were treated with gemcitabine alone $\left(300 \mathrm{mg} / \mathrm{m}^{2}\right)$ as a $30-\mathrm{min}-$ ute intravenous infusion once weekly during radiation therapy. Four to six weeks after the end date of chemo-radiotherapy, patients underwent preoperative evaluation, including computed tomography, positron emission tomography, and measurement of serum carbohydrate antigen 19-9 (CA19-9). If resection was deemed feasible, surgery was performed 1 to 2 weeks later. Demographic and clinical characteristics, including age, sex, cancer stage, were evaluated and analyzed.
Survival was estimated using the Kaplan-Meier method, with patients alive at the time of follow-up censored. Survival curves were constructed using Prism 5 (GraphPad software, La Jolla, CA, USA). Between-group differences in survival were assessed with the log-rank (Mantel-Cox) test.

\section{Comprehensive cancer panel}

Genomic DNA of patients was extracted from blood samples using QIAamp blood DNA mini kits (Qiagen, Valencia, CA, USA) and from formalin-fixed paraffin embedded (FFPE) endoscopic ultrasound (EUS)-guided biopsy samples, and surgically resected tissue using QIAamp DNA FFPE tissue kits (Qiagen). To prevent sequencing artifacts, DNA samples were treated with uracilDNA glycosylase prior to amplification. ${ }^{16,17}$ Targeted panel sequencing was performed with the Ion AmpliSeq Comprehensive

Table 1. Demographic and Clinical Characteristics of Patients with Pancreatic Ductal Adenocarcinoma

\begin{tabular}{|c|c|}
\hline Characteristic & Patient \\
\hline Total patients & 22 \\
\hline EUS specimens & 7 \\
\hline Surgically resected specimens & 22 \\
\hline \multicolumn{2}{|l|}{ Sex } \\
\hline Male & $12(54.5)$ \\
\hline Female & $10(45.5)$ \\
\hline Age at cancer diagnosis, yr & $67(51-76)$ \\
\hline \multicolumn{2}{|l|}{ Smoking history } \\
\hline Yes & 12 \\
\hline No & 10 \\
\hline Pack-years for smokers & 38 (10-98) \\
\hline Tumor size, mL & $2.7(0.6-5.8)$ \\
\hline \multicolumn{2}{|l|}{ Tumor location } \\
\hline Head & 15 \\
\hline Body and tail & 7 \\
\hline \multicolumn{2}{|l|}{ Endolymphatic tumor emboli } \\
\hline Absent & 14 \\
\hline Present & 8 \\
\hline \multicolumn{2}{|l|}{ Blood vessel invasion } \\
\hline Absent & 13 \\
\hline Present & 9 \\
\hline Pretreatment CA19-9, U/mL & $82(0-3,334)$ \\
\hline \multicolumn{2}{|l|}{ Adjuvant chemotherapy } \\
\hline Yes & 12 \\
\hline No & 10 \\
\hline Recurrence & 20 \\
\hline Death & 18 \\
\hline Follow-up time, mo & $20(5-59)$ \\
\hline
\end{tabular}

Data are presented as number (\%) or median (range). EUS, Endoscopic ultrasound; CA19-9, carbohydrate antigen 19-9. 
Cancer Panel covering 409 genes (Ion Torrent, Life Technologies, Carlsbad, CA, USA). Libraries were prepared for sequencing according to the manufacturer's instructions, and the quality of the libraries was determined using a 2100 Bioanalyzer (Agilent Technologies, Santa Clara, CA, USA). Sequencing was performed using the platform Nextseq 500 System platform, with $2 \times 151$ bp paired end sequencing runs (Illumina Inc., San Diego, CA, USA).

Next-generation sequencing data were generated from 22 tumor samples, seven biopsy samples, and 22 blood samples from the 22 patients with PDA. The cancer panel covering $1.3 \mathrm{Mbp}$ from 409 cancer-related genes produced about $16.4 \mathrm{M}$ reads per sample. Reads with low quality were excluded from further analyses. The targeted average coverage was greater than 1200x for tumor samples and 500x for germline DNA.

\section{Analysis of somatic mutations}

Sequencing reads for the 409 target genes were processed using CASAVA base calling software version 1.8.2 (Illumina, Hayward, CA, USA). The read length was $150 \mathrm{bp}$, with insert sizes of 125 to $175 \mathrm{bp}$. Sequence index, quality score from FASTQ and individual Phred scores were assessed for sequencing quality. Sequences were analyzed with Genome Analysis Toolkit version 3.3 (Broad Institute, Cambridge, MA, USA) ${ }^{18}$ after mapping with BWA tools. ${ }^{19,20}$ Somatic mutations were designated by MuTect and Strelka after comparing sequences in tumor tissues with their corresponding blood samples. ${ }^{21,22}$ Variants were annotated with SNPEff 4.1 (GRCh 37.75). The effects of variants were predicted with Polyphen2 and SIFT scores. ${ }^{23,24}$ Allelic fractions of somatic mutations were compared in sequences obtained from EUS-guided biopsy and surgically resected tumor samples. Mutations were visualized using OncoPrint and MutationMapper by cBioPortal. ${ }^{25,26}$

\section{RESULTS}

\section{Clinical characteristics of PDA patients and treatment outcomes}

The demographic features of the 22 patients with PDA are summarized in Table 1 . These 22 patients included 12 men and 10 women, of median age 67 years. Median follow-up time was 20 months (range, 5-59 months). Smoking history and tumor size were not significant predictors of poor prognosis, whereas lymphatic invasion, as determined by endolymphatic tumor emboli, was significantly associated with poor median survival (13 months vs 26 months, $p=0.0037$ ) (Supplementary Fig. 1).

\section{Somatic mutation pattern and related survival character- istics of 22 PDA patients}

Following the analysis of somatic mutations in these 22 patients, nonsense, missense, frameshift and splice site mutations were selected for further analyses because of the effects of these mutations on their encoded proteins. Four patients had a high mutation burden as shown in Fig. 1, averaging 1,545
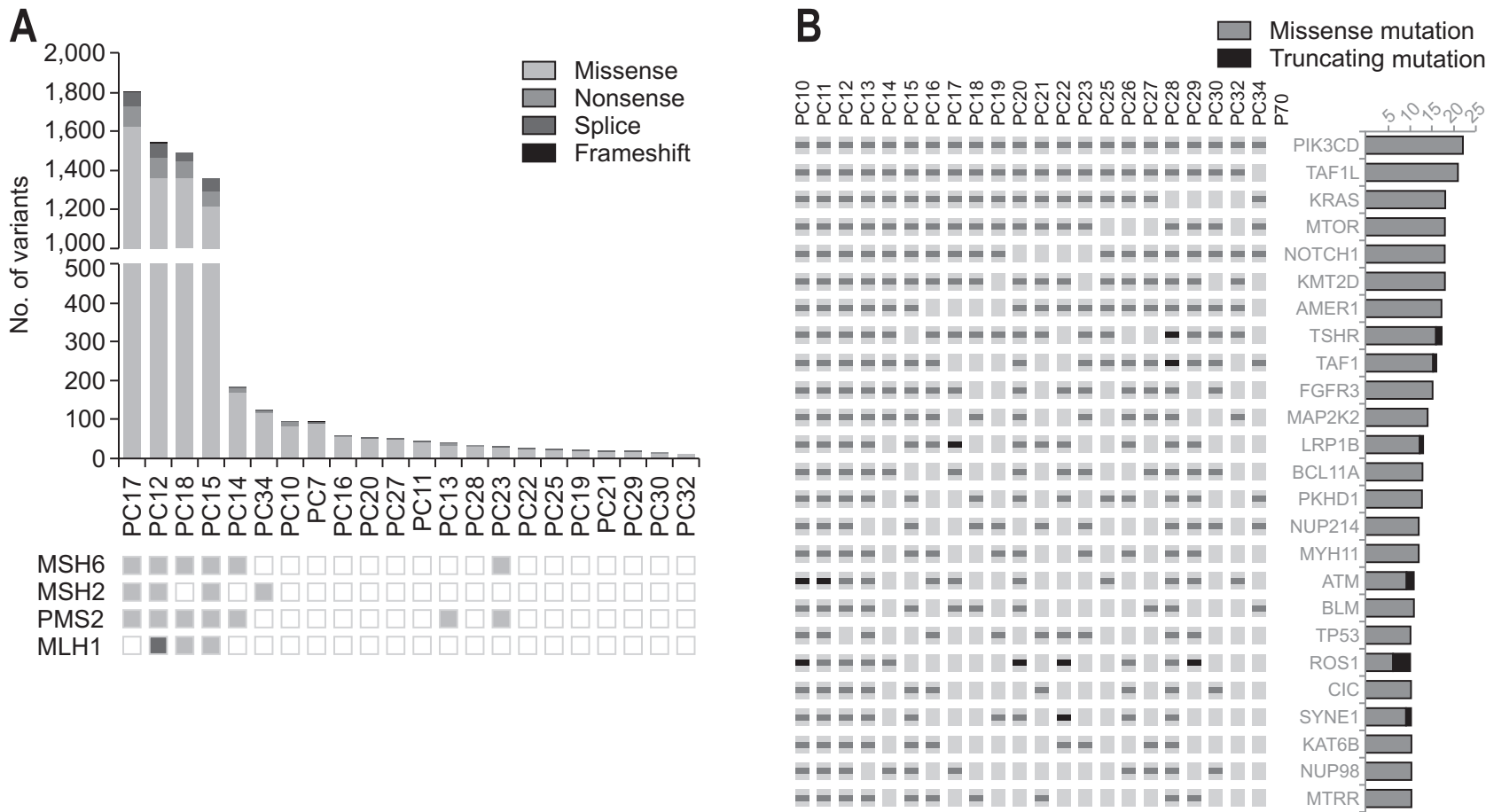

Fig. 1. Overall mutations from the cancer gene panel data of pancreatic ductal adenocarcinoma (PDA) patients. (A) Total number of variants in each of the 22 patients. Patients classified as hypermutated were enriched for mutations in mismatch repair genes. (B) Genes frequently altered in patients with PDA. The bar at the right shows the number of patients harboring alterations in each gene. 
nonsynonymous mutations per patient, whereas the other 18 patients had a mutation burden of 50.7 nonsynonymous mutations per patient. To determine whether high mutation burden was associated with mismatch repair genes, mutations in the MSH2, MSH6, MLH1, MLH3, POLE, EXO1, and PMS2 genes were examined. Assessments of the MLH1, MLH3, MSH2, and PMS2 genes showed that the four patients with the highest relative burden of mutations harbored somatic mutations in at least three of these genes (Fig. 1). However, high mutational load was not associated with overall survival (Supplementary Fig. 2).

KRAS mutations, frequently observed in PDA, were detected in 18 of the 22 tumors (82\%), with 17 having mutations in codon 12 and one in codon 61. PIK3CD, TAF1L, and MTOR were altered in most patients, whereas TP53 was mutated in 12 patients. Genes altered in more than 10 patients and their major mutations are also shown in Fig. 1. We also tested the effect of somatic mutations in TP53, KRAS, and ARID1A on clinical outcome. Survival curves indicated that median survival was shorter in patients harboring ARID1A mutations than wild type (14 months vs 23.5 months, $\mathrm{p}=0.05$ ) (Fig. 2). In contrast, TP53 and KRAS mutations were not associated with survival (Supplementary Fig. 2).

Highly mutated genes showing high frequency and multiple mutation sites are shown in Table 2. Gene set enrichment analyses showed that these highly mutated genes included those involved in chromatin modification, such as KMT2D, TAF1, ATM, and WHSC1. Members of the histone-lysine Nmethyltransferase 2 (KMT2) gene family, especially, KMT2D, KMT2A, and KMT2C, which are highly mutated in patients with mixed-lineage leukemia (MLL), were also highly mutated in our patients with PDA (Supplementary Fig. 3).

\section{Genetic diversity before /after treatment in seven pa- tients}

The somatic mutation patterns were compared in biopsy samples obtained at diagnosis of seven patients and in their surgically resected samples after treatment. Of these, three had resectable tumors at initial diagnosis. The remaining four had locally advanced cancer. Somatic mutations with a reduced allelic fraction $(<1.5$-fold) after compared to before treatment in more than three patients are shown in Table 3. The allelic fraction of KRAS codon 12 mutations was lower in surgically resected specimens than in EUS samples of six patients, and the allelic fraction of the codon 1020 mutation P1020A in the histone lysine methyltransferase gene WHSC1 was lower after than before treatment in four patients.

\section{DISCUSSION}

Pancreatic cancer is one of the most common causes of cancer deaths worldwide, with a very poor 5-year survival rate. Due to asymptomatic progress and a lack of effective screening markers, most patients are diagnosed at an advanced stage. Current chemotherapy regimens for patients with advanced pancreatic cancer frequently include gemcitabine, but treatment responses vary widely among patients, due to the molecular genetic diversity of individual tumors. ${ }^{27,28}$

This study analyzed the somatic mutations of 22 Korean patients with PDA using CCP. All patients received neoadjuvant treatment inside clinical trial. Four of these patients showed a hypermutated pattern, with more than 1,000 mutations in mismatch repair genes, a finding consistent with the mismatch repair deficiency and "mutator" phenotype of PDA. ${ }^{29}$ Mutation burden was unrelated to smoking history or overall survival in

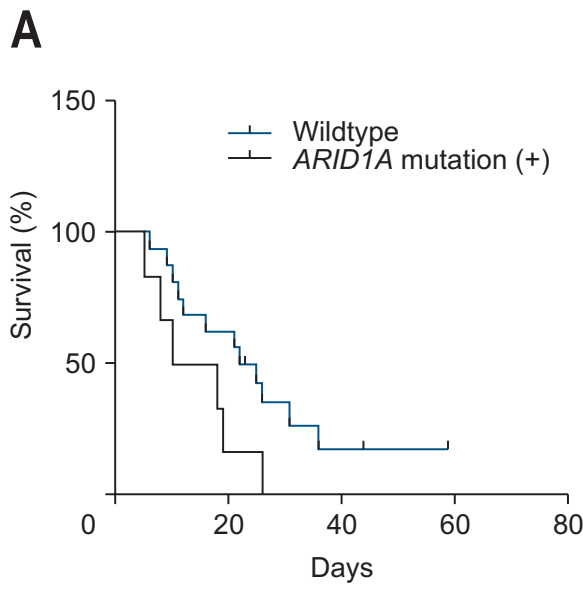

B

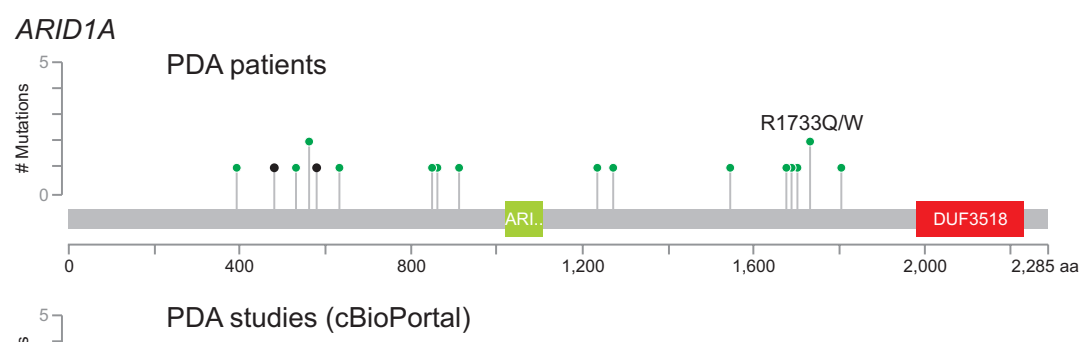

Fig. 2. ARID1A gene mutations and their association with disease outcome. (A) Kaplan-Meier analysis of overall survival in patients with and without ARID1A mutations. Median survival was shorter in patients harboring ARID1A mutations than in those with wildtype ARID1A (14 months vs 23.5 months, $\mathrm{p}=0.05$ ). (B) Comparison of ARID1A gene mutations detected in our patients and those in pancreatic ductal adenocarcinoma (PDA) patients listed in cBioPortal. Gray indicates missense mutations, and black indicates truncating mutations, including nonsense and frameshift mutations. 
Table 2. Genes Highly Mutated in Patients with Pancreatic Ductal Adenocarcinoma

\begin{tabular}{|c|c|c|c|c|}
\hline GSEA & Gene & Description & Total score* & Frequent mutant type \\
\hline \multirow{12}{*}{$\begin{array}{l}\text { Chromatin } \\
\text { modification }\end{array}$} & KMT2D & Lysine (K)-specific methyltransferase 2D & 131 & L449Q \\
\hline & KMT2A & Lysine (K)-specific methyltransferase 2A & 81 & \\
\hline & TRRAP & Transformation/transcription domain-associated protein & 78 & \\
\hline & KMT2C & Lysine (K)-specific methyltransferase 2C & 68 & \\
\hline & TAF1L & $\begin{array}{l}\text { TAF1 RNA polymerase II, TATA box binding protein (TBP)-associated factor, } \\
\text { 210kDa-like }\end{array}$ & 66 & R1243Q \\
\hline & TAF1 & $\begin{array}{l}\text { TAF1 RNA polymerase II, TATA box binding protein (TBP)-associated factor, } \\
250 \mathrm{kDa}\end{array}$ & 65 & \\
\hline & KDM5C & Lysine (K)-specific demethylase 5C & 47 & \\
\hline & KAT6B & $\mathrm{K}$ (lysine) acetyltransferase $6 \mathrm{~B}$ & 46 & \\
\hline & EP400 & E1A binding protein $\mathrm{p} 400$ & 43 & \\
\hline & TET2 & Tet methylcytosine dioxygenase 2 & 43 & \\
\hline & EP300 & E1A binding protein p300 & 42 & \\
\hline & WHSC1 & Wolf-Hirschhorn syndrome candidate 1 & 40 & \\
\hline \multirow{3}{*}{$\begin{array}{l}\text { Protein kinase } \\
\text { activity }\end{array}$} & MTOR & Mechanistic target of rapamycin (serine/threonine kinase) & 70 & T600I \\
\hline & IGF2R & Insulin-like growth factor 2 receptor & 58 & \\
\hline & ROS1 & ROS proto-oncogene 1 , receptor tyrosine kinase & 44 & W729* \\
\hline \multirow[t]{15}{*}{ Cell cycle } & DST & Dystonin & 71 & I761M \\
\hline & USP9X & Ubiquitin specific peptidase 9 , X-linked & 55 & $\mathrm{R} 882 \mathrm{C}$ \\
\hline & NUP98 & Nucleoporin 98kDa & 46 & \\
\hline & AKAP9 & A kinase (PRKA) anchor protein (yotiao) 9 & 45 & \\
\hline & APC & Adenomatous polyposis coli & 41 & \\
\hline & NUP214 & Nucleoporin $214 \mathrm{kDa}$ & 41 & \\
\hline & SYNE1 & Spectrin repeat containing, nuclear envelope 1 & 152 & L1632P \\
\hline & RNF213 & Ring finger protein 213 & 104 & \\
\hline & PKHD1 & Polycystic kidney and hepatic disease 1 & 74 & \\
\hline & LRP1B & Low density lipoprotein receptor-related protein 1B & 63 & I321S \\
\hline & FN1 & Fibronectin 1 & 61 & \\
\hline & PTPRT & Protein tyrosine phosphatase, receptor type, $\mathrm{T}$ & 57 & L1214P \\
\hline & CSMD3 & CUB and Sushi multiple domains 3 & 55 & \\
\hline & UBR5 & Ubiquitin protein ligase E3 component n-recognin 5 & 55 & \\
\hline & MYH11 & Myosin, heavy chain 11 , smooth muscle & 49 & T1546M \\
\hline
\end{tabular}

*Total score included total number of mutations located in the same gene in all patients. GSEA, gene set enrichment analysis.

this patient cohort. Because sample size is not large enough to ensure an adequate power to detect statistical significance, we cannot draw any firm conclusion.

Mutation profiling of patients with PDA has reported recurrent mutations in KRAS, TP53, SMAD4 and ARID1A, all of which have been associated with patient prognosis. ${ }^{10,29}$ Our results showed that mutations in ARID1A tended to be associated with poor prognosis, whereas mutations in KRAS and TP53 were not. Mutations in ARID1A present before treatment decreased or disappeared after treatment in two patients. These results provide further evidence suggesting that ARID1A mutations may be a prognostic marker in patients with PDA.

We also found that some frequently mutated genes were involved in chromatin modification. Members of the histone lysine methyltransferase KMT2 (MLL) family of genes, including KMT2D, KMT2C, and KMT2A, showed a high frequency of alterations, including nonsynonymous and nonsense mutations. Interestingly, mutations in MLL, MLL2, and MLL3 were closely associated with survival outcomes in patients with PDA, ${ }^{30}$ suggesting that MLL mutation status was an independent prognos- 
Table 3. Somatic Mutations with Decreased Allelic Fractions after Treatment in at Least 3 Patients

\begin{tabular}{|c|c|c|c|}
\hline Gene & Description & Protein change & Patient \\
\hline KRAS & Kirsten rat sarcoma viral oncogene homolog & G12* & 6 \\
\hline WHSC1 & Wolf-Hirschhorn syndrome candidate 1 & P1020A & 4 \\
\hline CDK6 & Cyclin-dependent kinase 6 & p.N284H & 3 \\
\hline DDB2 & Damage-specific DNA binding protein $2,48 \mathrm{kDa}$ & p.W54L & 3 \\
\hline EP300 & E1A binding protein $\mathrm{p} 300$ & p.G98A & 3 \\
\hline ERCC3 & Excision repair cross-complementation group 3 & p.V193L & 3 \\
\hline FBXW7 & F-box and WD repeat domain containing 7, E3 ubiquitin protein ligase & p.A105S & 3 \\
\hline FLT3 & Fms-related tyrosine kinase 3 & p.R655G & 3 \\
\hline KAT6A & $\mathrm{K}$ (lysine) acetyltransferase $6 \mathrm{~A}$ & p.M1389L & 3 \\
\hline KAT6B & K(lysine) acetyltransferase 6B & p.Q1513E & 3 \\
\hline KDR & Kinase insert domain receptor (a type III receptor tyrosine kinase) & p.C246S & 3 \\
\hline MMP2 & Matrix metallopeptidase 2 (gelatinase A, 72kDa gelatinase, $72 \mathrm{kDa}$ type IV collagenase) & p.E258Q & 3 \\
\hline PSIP1 & PC4 and SFRS1 interacting protein 1 & p.A168G & 3 \\
\hline TET2 & Tet methylcytosine dioxygenase 2 & p.M1789I & 3 \\
\hline XPA & Xeroderma pigmentosum, complementation group A & p.L226W & 3 \\
\hline ZNF521 & Zinc finger protein 521 & p.D25E & 3 \\
\hline
\end{tabular}

${ }^{*}$ G12 indicates various mutant types on codon 12 of the KRAS gene including G12D, G12V and G12S.

tic factor associated with survival. Our study could not confirm these results, as only three of 22 patients lacked mutations in the KTM2 genes.

Our comparison of somatic mutation patterns in EUS-guided biopsy specimens collected at initial diagnosis and surgical specimens collected after concurrent chemo-radiotherapy showed a reduction in the allelic fraction of mutations in KRAS and WHSC1 after treatment. Decreased KRAS mutant fraction after treatment suggests the favorable response of neoadjuvant therapy. Furthermore, we demonstrated the feasibility of EUSguided biopsy samples for mutation profiling that can also be compared with the resected tissues. However, this finding requires confirmation in future studies comparing paired samples from a larger nubmer of patients. Indeed, the primary limitation of our study was the small number of PDA patients. Moreover, clinical follow-up was relatively short. Further study and validation will be needed to determine the utility of the detected alterations in PDAs. Nevertheless, our study could determine that PDAs are genetically complex and that ARID1A mutations may be prognostic of survival.

\section{CONFLICTS OF INTEREST}

No potential conflict of interest relevant to this article was reported.

\section{ACKNOWLEDGEMENTS}

This work was supported by National Cancer Center Research Grant (1410681) and National Research Foundation of Korea
(NRF-2015R1A2A2A01005812) grant funded by the Korean government.

\section{AUTHOR CONTRIBUTIONS}

Study concept and design: K.A.Y., S.M.W., S.J.P. Data acquisition: K.A.Y., S.M.W., S.J.P. Data analysis and interpretation: K.A.Y., S.M.W., M.K.L., S.S.H., T.H.K., W.J.L., S.J.P. Drafting of the manuscript: K.A.Y., S.M.W. Statistical analysis: K.A.Y., M.K.L. Obtained funding: K.A.Y., S.J.P. Critical revision of the manuscript for important intellectual content: S.M.W., Y.H.K., S.Y.K., S.S.H., T.H.K., W.J.L., S.J.P. Administrative, technical, or material support: Y.H.K., S.Y.K., M.K.L., S.S.H., T.H.K., W.J.L., S.J.P. Study supervision: Y.H.K., S.Y.K., S.S.H., T.H.K., W.J.L., S.J.P.

\section{ORCID}

Kyong-Ah Yoon https://orcid.org/0000-0002-9823-7393

Sang Myung Woo https://orcid.org/0000-0003-3786-4403

Yun-Hee Kim https://orcid.org/0000-0001-7571-9958

Sun-Young Kong https://orcid.org/0000-0003-0620-4058

Min Kyoung Lee https://orcid.org/0000-0002-1090-456X

Sung-Sik Han https://orcid.org/0000-0001-7047-7961

Tae Hyun Kim https://orcid.org/0000-0001-8413-3385

Woo Jin Lee https://orcid.org/0000-0003-2115-6701

Sang-Jae Park https://orcid.org/0000-0001-5582-9420

\section{REFERENCES}

1. Jung KW, Won YJ, Oh CM, et al. Cancer statistics in Korea: in- 
cidence, mortality, survival, and prevalence in 2014. Cancer Res Treat 2017;49:292-305.

2. Oh CM, Won YJ, Jung KW, et al. Cancer statistics in Korea: incidence, mortality, survival, and prevalence in 2013. Cancer Res Treat 2016;48:436-450.

3. Ryan DP, Hong TS, Bardeesy N. Pancreatic adenocarcinoma. N Engl J Med 2014;371:1039-1049.

4. Von Hoff DD, Ervin T, Arena FP, et al. Increased survival in pancreatic cancer with nab-paclitaxel plus gemcitabine. N Engl J Med 2013;369:1691-1703.

5. Garrido-Laguna I, Hidalgo M. Pancreatic cancer: from state-ofthe-art treatments to promising novel therapies. Nat Rev Clin Oncol 2015;12:319-334.

6. Bournet B, Buscail C, Muscari F, Cordelier P, Buscail L. Targeting KRAS for diagnosis, prognosis, and treatment of pancreatic cancer: hopes and realities. Eur J Cancer 2016;54:75-83.

7. Kwon MJ, Jeon JY, Park HR, et al. Low frequency of KRAS mutation in pancreatic ductal adenocarcinomas in Korean patients and its prognostic value. Pancreas 2015;44:484-492.

8. Biankin AV, Waddell N, Kassahn KS, et al. Pancreatic cancer genomes reveal aberrations in axon guidance pathway genes. Nature 2012;491:399-405.

9. Cowley MJ, Chang DK, Pajic M, et al. Understanding pancreatic cancer genomes. J Hepatobiliary Pancreat Sci 2013;20:549-556.

10. Waddell N, Pajic M, Patch AM, et al. Whole genomes redefine the mutational landscape of pancreatic cancer. Nature 2015;518:495501.

11. Wood LD. Pancreatic cancer genomes: toward molecular subtyping and novel approaches to diagnosis and therapy. Mol Diagn Ther 2013;17:287-297

12. Buys SS, Sandbach JF, Gammon A, et al. A study of over 35,000 women with breast cancer tested with a 25-gene panel of hereditary cancer genes. Cancer 2017;123:1721-1730.

13. Malapelle U, Mayo de-Las-Casas C, Rocco D, et al. Development of a gene panel for next-generation sequencing of clinically relevant mutations in cell-free DNA from cancer patients. Br J Cancer 2017;116:802-810.

14. Rathi V, Wright G, Constantin D, et al. Clinical validation of the 50 gene AmpliSeq Cancer Panel V2 for use on a next generation sequencing platform using formalin fixed, paraffin embedded and fine needle aspiration tumour specimens. Pathology 2017;49:7582.

15. Woo SM, Kim MK, Joo J, et al. Induction chemotherapy with gemcitabine and cisplatin followed by simultaneous integrated boostintensity modulated radiotherapy with concurrent gemcitabine for locally advanced unresectable pancreatic cancer: results from a feasibility study. Cancer Res Treat 2017;49:1022-1032.

16. Do H, Dobrovic A. Dramatic reduction of sequence artefacts from DNA isolated from formalin-fixed cancer biopsies by treatment with uracil- DNA glycosylase. Oncotarget 2012;3:546-558.

17. Serizawa M, Yokota T, Hosokawa A, et al. The efficacy of uracil DNA glycosylase pretreatment in amplicon-based massively parallel sequencing with DNA extracted from archived formalinfixed paraffin-embedded esophageal cancer tissues. Cancer Genet 2015;208:415-427.

18. McKenna A, Hanna M, Banks E, et al. The Genome Analysis Toolkit: a MapReduce framework for analyzing next-generation DNA sequencing data. Genome Res 2010;20:1297-1303.

19. Li H, Durbin R. Fast and accurate short read alignment with Burrows-Wheeler transform. Bioinformatics 2009;25:1754-1760.

20. Li H, Durbin R. Fast and accurate long-read alignment with Burrows-Wheeler transform. Bioinformatics 2010;26:589-595.

21. Cibulskis K, Lawrence MS, Carter SL, et al. Sensitive detection of somatic point mutations in impure and heterogeneous cancer samples. Nat Biotechnol 2013;31:213-219.

22. Saunders CT, Wong WS, Swamy S, Becq J, Murray LJ, Cheetham RK. Strelka: accurate somatic small-variant calling from sequenced tumor-normal sample pairs. Bioinformatics 2012;28:1811-1817.

23. Sim NL, Kumar P, Hu J, Henikoff S, Schneider G, Ng PC. SIFT web server: predicting effects of amino acid substitutions on proteins. Nucleic Acids Res 2012;40(Web Server issue):W452-W457.

24. Adzhubei I, Jordan DM, Sunyaev SR. Predicting functional effect of human missense mutations using PolyPhen-2. Curr Protoc Hum Genet 2013;76:7.20.1-7.20.41.

25. Cerami E, Gao J, Dogrusoz U, et al. The cBio cancer genomics portal: an open platform for exploring multidimensional cancer genomics data. Cancer Discov 2012;2:401-404.

26. Gao J, Aksoy BA, Dogrusoz U, et al. Integrative analysis of complex cancer genomics and clinical profiles using the cBioPortal. Sci Signal 2013;6:pl1.

27. Turner NC, Reis-Filho JS. Genetic heterogeneity and cancer drug resistance. Lancet Oncol 2012;13:e178-e185.

28. Wang Z, Li Y, Ahmad A, et al. Pancreatic cancer: understanding and overcoming chemoresistance. Nat Rev Gastroenterol Hepatol 2011;8:27-33.

29. Witkiewicz AK, McMillan EA, Balaji U, et al. Whole-exome sequencing of pancreatic cancer defines genetic diversity and therapeutic targets. Nat Commun 2015;6:6744.

30. Sausen M, Phallen J, Adleff V, et al. Clinical implications of genomic alterations in the tumour and circulation of pancreatic cancer patients. Nat Commun 2015;6:7686. 